\title{
Basics of Blenderized Tube Feeds: A Primer for Pediatric Primary Care Clinicians
}

\author{
Judy-April Oparaji ${ }^{\mathrm{a}}$, Thomas Sferra ${ }^{\mathrm{b}}$, Senthilkumar Sankararaman ${ }^{\mathrm{b}, \mathrm{c}}$
}

\begin{abstract}
Blenderized tube feeding (BTF) is defined as the use of blended foods and liquids given directly via the feeding tube. This form of tube feeding lost popularity with the introduction of commercial enteral formulas in the 1970s; however, society's recent focus on more natural foods has led to a reemerged interest in blenderized feeds. BTF is particularly popular among the pediatric population for a variety of reasons. Many patients and families choose BTF because of its perceived health benefits, intolerance to commercial feeding formulas, or psychosocial reasons. Despite its increasing use, the current literature on the prevalence, safety and outcome of BTF is limited. In this review, we discuss the potential benefits and drawbacks of blenderized tube feeds. We also review clinical application pearls for pediatric primary care clinicians. It is important for these clinicians to have a basic understanding of blenderized formulas in order to support families that are interested in this reemerging food practice.
\end{abstract}

Keywords: Nutrition; Enteral nutrition; Pediatrics

\section{Introduction}

Enteral nutritional support, commonly known as tube feeding, is the process of delivering nutrients via tube through the nose, mouth, or stoma into the gastrointestinal (GI) tract when oral intake cannot meet the energy and metabolic demands of an individual. Tube feedings most often involve commercial cow milk protein-based or amino acid formulas; however, with society's new focus on whole foods, there has been a resurgence of interest in utilizing more natural foods through the feeding tube.

Blenderized tube feeding (BTF) is defined as the use of blended foods and liquids given directly via the feeding tube.

Manuscript submitted May 16, 2019, accepted May 31, 2019

${ }^{\text {aW }}$ omack Army Medical Center, Fort Bragg, NC, USA

bDivision of Pediatric Gastroenterology \& Nutrition, UH Rainbow Babies \& Children's Hospital, Cleveland, OH, USA

${ }^{\mathrm{c} C}$ Corresponding Author: Senthilkumar Sankararaman, Division of Pediatric Gastroenterology, Hepatology \& Nutrition, UH Rainbow Babies \& Children's Hospital, 11100 Euclid Ave., Cleveland, OH 44106, USA. Email: Senthilkumar.Sankararaman@UHhospitals.org

doi: https://doi.org/10.14740/gr1192
Blenderized feeds could refer to homemade BTF, commercial formula mixed with pureed baby food or any of the variety of commercially available ready to use BTFs (Table 1) [1]. Other commonly used terms to refer to this type of diet and feeding are blended diet, blenderized feeding and homemade or commercial blended formula. Many patients and families choose BTF because of the perceived health benefits, intolerance to commercial feeding formulas, or psychosocial reasons (e.g. desire for "real" food). There are potential drawbacks to the use of BTF including the increased risk of bacterial contamination, more frequently clogged tubes and greater labor intensity in preparation of the feeding. Families may use or intend to use blenderized foods for partial, supplemental or complete nutrition support [2]. A 2016 survey of patients requiring home enteral nutrition found that $89.6 \%$ of pediatric patients used BTF in varying amounts [3]. Despite its increasing use, the current literature on the prevalence, safety and outcomes of BTF remains limited.

\section{History}

Enteral feeding dates back more than 3,500 years ago to the ancient Egyptians who attempted rectal administration of wine, milk and grain broths [4]. In the 1950s and 1960s, real blenderized foods once widely prevalent in hospitals in North America and Europe went out of favor with the introduction of commercial dairy-based formulas. By the 1970s, commercial formulas were widely adopted as complete nutrition that was sterile, easy to administer and less expensive than blended formulas made in hospital kitchens [2]. However, BTF use remained prevalent in resource limited countries and remote settings where the availability of commercial formulas was limited either due to cost or logistics $[5,6]$.

\section{Perceived Benefits}

\section{Psychosocial}

One of the most common reasons that families want to use BTF is to normalize the tube feeding experience. By offering foods the entire family eats, children requiring enteral nutrition can be included in family meals. In addition, meals can be prepared to meet specific diet needs and preferences or to omit certain food allergens [7]. 
Table 1. Patient/Clinician Resources

\begin{tabular}{ll}
\hline $\begin{array}{l}\text { Oley Foundation (non-profit educational resource } \\
\text { for patients on enteral or parenteral nutrition) }\end{array}$ & https://oley.org/ \\
Food safety guidelines & www.homefoodsafety.org \\
& www.foodsafety.gov \\
Sample BTF recipes & www.ginutrition.virginia.edu \\
& www.foodfortubies.org \\
& www.mealtimenotions.com \\
\hline Ready-to-use BTF* & Compleat - nestlehealthscience.us \\
& Kate Farms - katefarms.com \\
& Liquid Hope, Nourish - functionalformularies.com \\
& Pediasure Harvest - abbottstore.com \\
& Real Food Blends - realfoodblends.com \\
\hline
\end{tabular}

*Commercial BTF hang time varies from 8 to $12 \mathrm{~h}$, based on individual manufacturer's recommendations. Any unused formula should be discarded after $24 \mathrm{~h}$ [1]. BTF: blenderized tube feeding.

\section{Gut health}

Advocates of BTF argue that whole foods may benefit the gut microbiota. Further research is being done on the benefits of prebiotics and phytonutrients that are found in fruits and vegetables that can be a part of BTF [1]. In one of the few prospective studies on gut health and BTF, stool bacterial diversity, monitored by rDNA-based sequencing, was improved upon a transition from commercial formula to BTF in a small group of medically complex pediatric patients [8].

\section{Feeding tolerance}

Another common reason families request BTF is feeding intolerance with commercial formulas. Many parents report improved growth and fewer symptoms of feeding intolerance (reflux, gagging and constipation) after switching to BTF [9]. In a study of 33 children who were post Nissen fundoplication, $52 \%$ of parents reported a $76-100 \%$ improvement in postprandial gagging and retching after the initiation of a blended diet [10]. Despite many anecdotal benefits of BTF, definitive data are lacking and more research is needed to support these claims.

\section{Drawbacks}

\section{Bacterial contamination}

Bacterial contamination of the blenderized food is one of the biggest concerns with BTF and has been frequently reported in the literature; however, none of these studies have shown a clinical correlation with acute infection in patients [11-13]. Contamination of BTF can occur during preparation or handling. Proper hand hygiene and food safety practices must be reinforced when preparing and administering the feedings. Home- made BTF should not be left at room temperature for more than $2 \mathrm{~h}$. Many schools will not allow staff to give homemade BTF because of food safety concerns. Some families use homemade BTF at home and a commercial BTF at school [7, 14].

\section{Mechanical issues}

The increased viscosity of blenderized foods can clog feeding tubes [15]. If homemade BTF is used, it must be evenly blended. Larger feeding tubes of at least $14 \mathrm{Fr}$ also decrease the risk of clogging. Over time, some oils in homemade BTF can degrade the plastic that feeding tubes are made from. Flushing the tube with water after every feeding is essential to reduce the risks of clogging and degrading the feeding tubes.

\section{Nutritional deficiencies}

There are significant differences and inconsistencies in BTF preparation among care givers [9]. In infants and young children with higher energy demands, nutritional inadequacy from unsupervised BTF may cause delayed growth from both macroand micronutrient deficiencies [16]. Some commercially available blenderized formulas are not meant to provide all nutrient dietary reference intakes (DRIs) or daily calorie and protein requirements. In addition, it is important to ensure appropriate electrolyte content and maintenance fluid needs are met.

\section{Cost}

A study done at Cincinnati Children's Hospital estimated that the average daily cost of blended foods was $\$ 6.20$ versus $\$ 8.00$ for commercial formulas [10]. Despite the lower direct costs of BTF, baby food, groceries, commercial blenderized formulas and high-grade blenders are not covered by most insurance companies. Medicare and Medicaid require a documented al- 
Table 2. Good Candidates for BTF

\author{
Medically stable; stable growth \\ $\geq 14$ Fr feeding tube \\ Bolus feeds \\ Motivated family \\ Access to knowledgeable medical providers \\ BTF: blenderized tube feeding. \\ Table 3. Potential Contraindications \\ Acute illness or immunosuppression (greater risk of infection from contaminated food) \\ Fluid restrictions (may be difficult to meet nutrient needs) \\ Continuous feedings (requires formula to be unrefrigerated for several hours)
}

Adequate resources and equipment (high-grade blender, refrigerator, electricity, access to clean water and food, storage containers)

lergy or intolerance to commercial semi-synthetic formulas to approve coverage for commercial BTF [1]. Collaboration with social workers can help families receive the proper assistance.

\section{Patient Selection}

Tables 2 and 3 list criteria to consider when selecting the appropriate patient for BTF. A medical team's support and education are imperative. The use of BTF is more time-intensive than commercial formulas, so a family must be motivated to succeed. Families that are discouraged by time constraints could be offered ready-to-feed commercial BTF as an alternative. Commercial BTF also may be a safer option than homemade BTF for patients at increased risk of infection.

\section{Role of Primary Care Clinicians}

A recent survey found that only $50 \%$ of patients on BTF use a healthcare provider to help create nutritionally complete recipes [17]. Given the increasing popularity and use of BTF, it is important for pediatric clinicians to gain familiarity and knowledge of BTF to provide optimal nutritional support for their patients. Hence, if a family expresses interest, or is already providing unsupervised BTF, the clinician should provide further guidance including discussions as to the potential risks and benefits of this form of nutrition. If the decision is to begin or continue to use BTF, the clinician should consult with pediatric gastroenterologists and registered dietitians (RDs) who have expertise in BTF. Table 1 lists some basic resources for clinicians and families to learn about BTF and food safety.

\section{Conclusion}

Primary care clinicians must have a basic understanding of blenderized formulas in order to support families that are interested in this reemerging food practice. A multidisciplinary nutrition support team with familiarity with BTF management is a requisite to support families. At a minimum, this team includes a pediatric gastroenterologist or other physician with specific expertise in nutrition and an RD.

\section{Acknowledgments}

Thank you to Nicole Lidyard RDN, LD for her review of the manuscript.

\section{Financial Disclosure}

Dr. Sferra is a member of a scientific advisory committee and local principal investigator for a Merck \& Co., Inc. pharmaceutical sponsored trial. Drs. Oparaji and Sankararaman have disclosed no financial relationships relevant to this article. This commentary does not contain a discussion of an unapproved/ investigative use of a commercial product/device.

\section{Conflict of Interest}

None to declare.

\section{Author Contributions}

All listed authors made substantial contributions to the conception, drafting and revisions of this manuscript. All authors have approved the final version of this manuscript and agree to be accountable for all aspects of the work.

\section{References}

1. Martin K, Gardner G. Home enteral nutrition: updates, trends, and challenges. Nutr Clin Pract. 2017;32(6):712- 
721.

2. Parrish C. Blenderized tube feeding: suggested guidelines to clinicians. Pract Gastroenterol. 2014;136:58-66.

3. Epp L, Lammert L, Vallumsetla N, Hurt RT, Mundi MS. Use of blenderized tube feeding in adult and pediatric home enteral nutrition patients. Nutr Clin Pract. 2017;32(2):201-205.

4. Harkness L. The history of enteral nutrition therapy: from raw eggs and nasal tubes to purified amino acids and early postoperative jejunal delivery. J Am Diet Assoc. 2002;102(3):399-404.

5. Frizzi JD, Ray PD, Raff JB. Enteral nutrition by a forward surgical team in Afghanistan. South Med J. 2005;98(3):273-278.

6. Borghi R, Dutra Araujo T, Airoldi Vieira RI, Theodoro de Souza T, Waitzberg DL. ILSI Task Force on enteral nutrition; estimated composition and costs of blenderized diets. Nutr Hosp. 2013;28(6):2033-2038.

7. Bobo E. Reemergence of blenderized tube feedings: exploring the evidence. Nutr Clin Pract. 2016;31(6):730735.

8. Gallagher K, Flint A, Mouzaki M, Carpenter A, Haliburton B, Bannister L, Norgrove H, et al. Blenderized enteral nutrition diet study: feasibility, clinical, and microbiome outcomes of providing blenderized feeds through a gastric tube in a medically complex pediatric population. JPEN J Parenter Enteral Nutr. 2018;42(6):1046-1060.

9. Johnson TW, Spurlock AL, Epp L, Hurt RT, Mundi MS. Reemergence of blended tube feeding and parent's reported experiences in their tube fed children. J Altern Com- plement Med. 2018;24(4):369-373.

10. Pentiuk S, O'Flaherty T, Santoro K, Willging P, Kaul A. Pureed by gastrostomy tube diet improves gagging and retching in children with fundoplication. JPEN J Parenter Enteral Nutr. 2011;35(3):375-379.

11. Jalali M, Sabzghabaee AM, Badri SS, Soltani HA, Maracy MR. Bacterial contamination of hospital-prepared enteral tube feeding formulas in Isfahan, Iran. J Res Med Sci. 2009;14(3):149-156.

12. Sullivan MM, Sorreda-Esguerra P, Santos EE, Platon BG, Castro CG, Idrisalman ER, Chen NR, et al. Bacterial contamination of blenderized whole food and commercial enteral tube feedings in the Philippines. J Hosp Infect. 2001;49(4):268-273.

13. Mokhalalati JK, Druyan ME, Shott SB, Comer GM. Microbial, nutritional and physical quality of commercial and hospital prepared tube feedings in Saudi Arabia. Saudi Med J. 2004;25(3):331-341.

14. Novak P, Wilson KE, Ausderau K, Cullinane D. The Use of blenderized tube feedings. ICAN Infant, Child, Adolesc Nutr. 2009;1(1):21-23.

15. Mortensen M. Blenderized Tube Feeding Clinical Perspectives on Homemade Tube Feeding. Natl (Pediatric Nutr Pract Group) PNPG Post. 2006;17(1):1-4.

16. Johnson TW, Milton DL, Johnson K, et al. Comparison of microbial growth between commercial formula and blenderized food for tube feeding. Nutr Clin Pract. 2019;34(2):257-263.

17. Epp L. Blenderized feeding options - the sky's the limit. Pract Gastroenterol. 2018;(June). 